\title{
A COUNTEREXAMPLE TO THE GENERALIZED BANACH THEOREM
}

WLODZIMIERZ BZYL

\begin{abstract}
We show that it is consistent that the family of Borel maps of class 2 differs from the family of pointwise limits of Borel maps of class 1 . This gives an answer to a question raised by W. G. Fleissner.
\end{abstract}

1. Let $X, Y$ be metric spaces. For $0 \leqslant \alpha \leqslant \omega_{1}$, denote by $\Sigma_{\alpha} X$ the family of sets in $X$ of additive class $\alpha$. Define the Borel classes by

$$
\psi_{\alpha}(X, Y)=\left\{f: X \rightarrow Y: \forall\left(G \in \Sigma_{0} Y\right) f^{-1}[G] \in \Sigma_{\alpha} X\right\}
$$

and the Banach classes for $1 \leqslant \alpha \leqslant \omega$, by

$$
\phi_{1}^{*}(X, Y)=\psi_{1}(X, Y)
$$

and

$$
\begin{aligned}
& \phi_{\alpha}^{*}(X, Y)=\text { family of all limits of pointwise convergent sequences } \\
& \text { of maps from } \bigcup_{\beta<\alpha} \phi_{\beta}^{*}(X, Y) \quad(\alpha>1) .
\end{aligned}
$$

Correspondence between the Borel classes and the Banach classes is expressed by

Banach TheOREM. Let $Y$ be a separable metric space. Then $\phi_{\alpha}^{*}(X, Y)=\psi_{\alpha}(X, Y)$ or $\psi_{\alpha+1}(X, Y)$ according as $\alpha$ is finite or infinite. ${ }^{1}$

Recall that the inclusion $\phi_{\alpha}^{*}(X, Y) \subseteq \psi_{\alpha}(X, Y)$ or $\psi_{\alpha+1}(X, Y)$, according as $\alpha$ is finite or infinite, holds for any metric space $Y$.

Recently W. G. Fleissner $\left[\mathbf{F}_{1}\right]$ introduced an axiom called Proposition P. He proved that Proposition $\mathrm{P}$ is consistent, assuming that $\mathrm{ZFC}+$ 'there exists a supercompact cardinal' is consistent and that it implies, among other things, the Banach Theorem for any metric space $Y$. Later Fleissner, Hansell and Junnila [FHJ] proved that Proposition $\mathbf{P}$ is also implied by the Product Measure Extension Axiom.

In $\left[\mathbf{F}_{1}\right]$ the author asks whether it is consistent that $\phi_{2}^{*}(X, Y) \neq \psi_{2}(X, Y)$. In this note we show how the well-known Miller Theorem $[\mathbf{M}]$ can be used to give an affirmative answer to this question.

2. Recall that a $Q$ set is an uncountable subset $X$ of the real line $\mathbf{R}$ such that every subset of $X$ belongs to $\Sigma_{1} X$. It follows from the Miller Theorem (cf. [ $\mathbf{F}_{2}$, Theorem 23]) that the following proposition is consistent with ZFC.

Received by the edtiors November 9, 1982.

1980 Mathematics Subject Classification. Primary 54H05; Secondary 04A15.

${ }^{1}$ For more details about $\phi^{*}, \psi$ and the Banach Theorem, see [H]. 
Proposition. There is a subset $X$ of $\mathbf{R}$ with cardinality $\omega_{3}$ such that every subset of $X$ belongs to $\Sigma_{2} X$, but every $Q$ set contained in $X$ has cardinality $<\omega_{3}$.

THEOREM. If $|X|$ is the set $X$ with discrete metric, then $f \in \psi_{2}(X,|X|) \backslash \phi_{2}^{*}(X,|X|)$ for every injection from $X$ to $|X|$.

Proof. Clearly $f \in \psi_{2}(X,|X|)$. Suppose $f \in \phi_{2}^{*}(X,|X|)$. Then there is a sequence $\left\{f_{n}: n \in \omega\right\} \subseteq \psi_{1}(X,|X|)$ such that $f$ is the pointwise limit of $f_{n}$. Put $A_{n}=\{x \in X$ : $\left.f_{n}(x)=f(x)\right\}$. Since $X=\cup\left\{A_{n}: n \in \omega\right\}$ there is $n_{0} \in \omega$ such that the cardinality of $A_{n_{0}}$ is $\omega_{3}$. This implies $A_{n_{0}}$ is not a $Q$ set. Hence, since $f \mid A_{n_{0}}$ is an injection, $f \mid A_{n_{0}} \notin \psi_{1}\left(A_{n_{0}},|X|\right)$. On the other hand, $f_{n_{0}} \mid A_{n_{0}} \in \psi_{1}\left(A_{n_{0}},|X|\right)$ and $f_{n_{0}} \mid A_{n_{0}}=$ $f \mid A_{n_{0}}$, contradiction.

REMARK 1. Similarly, Miller's Theorem implies that for any nonlimit $\alpha>1$ there is a set $X_{\alpha}$ such that $\phi_{\alpha}^{*}\left(X_{\alpha},\left|X_{\alpha}\right|\right) \neq \psi_{\alpha}\left(X_{\alpha},\left|X_{\alpha}\right|\right)$ or $\psi_{\alpha+1}\left(X_{\alpha},\left|X_{\alpha}\right|\right)$ according as $\alpha$ is finite or infinite.

REMARK 2. Fleissner proved in $\left[\mathbf{F}_{1}\right]$ that under Proposition $\mathbf{P}$ for every function $f$ from $\psi_{1}(X, Y)$, where $X$ and $Y$ are arbitrary metric and Banach spaces, respectively, there is a residual set $T$ in $X$ such that $f \mid T$ is continuous and he raised the question as to whether 'residual' can be replaced by 'dense'. Observe, however, that if $f$ is a function from the rationals to $\mathbf{R}$ which has a discrete image, then $f \in \psi_{1}(\mathbf{Q}, \mathbf{R})$ and $f \mid T$ is not continuous for any dense subset $T$ of $\mathbf{Q}$.

\section{REFERENCES}

[F $\left.\mathbf{F}_{1}\right]$ W. G. Fleissner, An axiom for nonseparable Borel theory, Trans. Amer. Math. Soc. 251 (1979), 309-328.

$\left[\mathbf{F}_{\mathbf{2}}\right]$ _ Current research on $Q$ sets, Janos Bolyai Colloq. on Topology, Budapest, 1978.

[FHJ] W. G. Fleissner, R. W. Hansell and H. J. K. Junnila, PMEA implies Proposition P, Topology Appl. 13 (1982), 255-262.

[H] R. W. Hansell, On Borel mappings and Baire functions, Trans. Amer. Math. Soc. 194 (1974), 195-211.

[M] A. W. Miller, On the length of Borel hierarchies, Ann. Math. Logic 16 (1979), 233-267.

Department of Mathematics, Gdańsk University, Wita Stwosza 57, 80-952 Gdańsk, Poland 